\title{
Sustained enhancements in inhibitory control depend primarily on the reinforcement of fronto-basal anatomical connectivity
}

\author{
Camille Chavan $^{1} \cdot$ Michael Mouthon $^{1} \cdot$ Marie Simonet $^{1} \cdot$ Henri-Marcel Hoogewoud $^{2}$.

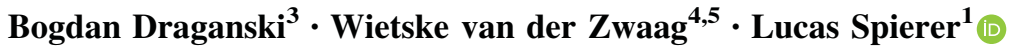

\begin{abstract}
What are the neurophysiological determinants of sustained supra-normal inhibitory control performance? We addressed this question by coupling multimodal neuroimaging and behavioral investigations of experts in fencing who underwent more than $20,000 \mathrm{~h}$ of inhibitory control training over 15 years. The superior control of the experts manifested behaviorally as a speeding-up of inhibition processes during a Go/NoGo task and was accompanied by changes in bilateral inferior frontal white matter microstructure. In the expert group, inhibition performance correlated positively with the fractional anisotropy (FA) of white matter tracts projecting to the basal ganglia, and the total training load with the FA in supplementary motor areas. Critically, the experts showed no changes in grey matter volume or in the functional organization of the fronto-basal inhibitory control network. The fencers' performance and neural activity during a 2-back working memory task did not differ from those of the controls,
\end{abstract}

Camille Chavan, Michael Mouthon contributed equally to this study.

Lucas Spierer

lucas.spierer@unifr.ch

1 Neurology Unit, Medicine Department, Faculty of Sciences, University of Fribourg, Chemin du Musée 5, 1700 Fribourg, Switzerland

2 Department of Radiology, Cantonal Hospital of Fribourg, Fribourg, Switzerland

3 LREN, Department of Clinical Neurosciences, Vaudois Hospital University Center, University of Lausanne, Lausanne, Switzerland

4 Center for Biomedical Imaging, École Polytechnique Fédérale de Lausanne, Lausanne, Switzerland

5 Spinoza Centre for Neuroimaging, Amsterdam, The Netherlands ensuring that their expertise was specific to inhibitory control. Our results indicate that while phasic changes in the patterns of neural activity and grey matter architecture accompany inhibitory control improvement after short- to medium- term training, long-lasting inhibitory control improvements primarily depend on the reinforcement of fronto-basal structural connectivity.

Keywords Inhibitory control · Plasticity - Training · Tract-based spatial statistics

\section{Introduction}

Inhibitory control (IC) essentially consists of inhibiting unwanted thoughts, emotions, or actions, and is mostly supported by a domain-general fronto-basal brain network (Aron et al. 2014).

Current literature reports that short- to medium-term training of IC improves the speed of inhibition processes and is associated with decreases in neural activity within the inferior frontal gyrus (Chavan et al. 2015; Manuel et al. 2013; Spierer et al. 2013; Hartmann et al. 2015), as well as to change in grey and white matter in the same areas (Chavan et al. 2015).

However, the neural mechanisms supporting sustained improvement in IC after very long-term training remain unknown. We addressed this question by investigating the anatomic and functional correlates of IC in an expert population who underwent years of intensive IC training. Elite fencers represent a highly suitable model for IC because this sport relies predominantly on IC (Roi and Bianchedi 2008; Di Russo et al. 2006). Opponents' feints and counter-feints at the core of fencing are indeed conflict situations in which fast suppressions of planned and 
ongoing actions are required. Together with the fact that fencers' training involve systematic practice of $\mathrm{Go} / \mathrm{NoGo}$ tasks, the key involvement of IC in fencers is supported by findings for superior IC proficiency (Chan et al. 2011; Di Russo et al. 2006; Taddei et al. 2012) and enhanced inhibition-related prefrontal P3 event-related potential components in fencers during inhibition tasks under controlled conditions (Di Russo et al. 2006; Taddei et al. 2012).

Based on (1) the central role of IC in fencing; (2) previous longitudinal investigations of IC training suggesting that improvements are achieved via a speeding up of inhibitory processes; and (3) evidence that the speed of neurocognitive processes depends on white matter microstructure properties (Tuch et al. 2005); we hypothesize that elite fencers who underwent an extensive IC training (in the present study $>20,000 \mathrm{~h}$ of training over $>15$ years), would primarily show white matter changes of the fronto-basal IC network, with only limited or absent functional and grey matter changes because of their putatively secondary role in the speed of inhibition processes (e.g., Waxman 1980).

To test these hypotheses, we used a cross-sectional experimental design comparing IC performance, as well as multimodal magnetic resonance imaging of neural activity and brain anatomy organization of the IC network between 19 world-level elite fencers and 18 age-matched, non-athlete control participants. A control 2-back task was used to test if the fencers' expertise was specific to inhibitory control.

\section{Materials and methods}

\section{Participants}

A total of 37 healthy volunteers participated in this study. The experts group included 19 male world-level elite fencers aged $27.3 \pm 0.6$ years (mean \pm SEM), four left-handed (Oldfield 1971). The elite fencers were selected with the criterion of having a world-level, which in our final population corresponded to a total of mean \pm SD $24,000 \pm 6,000 \mathrm{~h}$ of practice over $17.2 \pm 1.8$ years.

Participants of the control, non-athlete group included 18 participants from our previous study on medium-term IC training (Chavan et al. 2015, pre-training session; mean age \pm SEM : $25.1 \pm 0.7$ years; range: $22-32,8$ male, all right handed).

Each participant provided written, informed consent to participate in the study. No participant had a history of neurological or psychiatric disease. All procedures were approved by our local ethics committee.

To prevent confounds due to handedness, we included only right-handed participants in the functional magnetic resonance imaging (fMRI), voxel-based morphometry (VBM) and diffusion tensor imaging (DTI) analyses.

In the analyses, we eventually considered: For the behavior, 19 experts and 18 controls; for the fMRI, 14 experts (exclusion of 4 left-handed and 1 with problem during the fMRI scanning) and 18 controls; for the VBM: 15 experts (exclusion of 4 left-handed) and 18 controls; for the tract-based spatial statistics analysis (TBSS): 14 experts (exclusion of 4 left-handed and 1 with technical problems during MRI scanning) and 18 controls (inspection of the fractional anisotropy (FA) data revealed a bad quality probably due to movement (signal outside the $0-1$ range) for one of the control participant previously included in the fMRI and VBM analysis. To avoid losing statistical power, the data of this participant were replaced by those of a participant excluded from the fMRI and VBM analyses due to excessive movement during the fMRI, but who showed no head movements during the DTI acquisition.

\section{Procedures and tasks}

The procedures and tasks were the same as in Chavan et al. (2015), we report only the essential here.

\section{Go/NoGo inhibitory control task}

Functional MRI was recorded while the participants performed a Go/NoGo and a 2-back task to, respectively, assess IC and a task involving common components (sustained attention, alertness, flexibility ...) but not inhibition. In the Go/NoGo task, five consonants and four vowels were sequentially presented. Each trial started with a 1200-2200 ms fixation cross. Then, a letter was presented for $500 \mathrm{~ms}$ (in a pseudo-randomized order) and a response window open for max $1700 \mathrm{~ms}$. Each block consisted of 80 trials and the whole Go/NoGo task included 5 blocks of 3 min separated by $30 \mathrm{~s}$ of rest periods.

Participants were instructed to press with their right index finger on the button of the response box as fast as possible to each letter except the " $X$ ". The letter " $X$ " was the NoGo stimulus to which participants should inhibit the motor response. The stimulus probability was 0.3 for the NoGo stimulus and 0.7 for the Go stimulus.

\section{2-Back task}

In the control 2-back task, we used the same procedure and parameters as for the Go/NoGo task except that the task was not speeded, each letter had the same probability of presentation (0.1) and participants were instructed to indicate whether the second-last presented letter was a 
consonant or a vowel when they saw the letter " $\mathrm{X}$ ". Participants performed four blocks.

Tapping task

At the end of the Go/NoGo and of the 2-back task, we recorded BOLD responses during a tapping block, which was used to isolate motor brain activity related to the button press (see the functional magnetic resonance imaging section). Participants had to press a button each time a picture of a hand appeared on the screen (30 times for $500 \mathrm{~ms}$ over a period of $67 \mathrm{~s}$, with the same inter-trial interval as for the Go/NoGo and the 2-back tasks).

All stimulus delivery and response recording were controlled using E-Prime 2.0 software.

\section{Data acquisition, preprocessing and analyses}

\section{Behavioral analyses}

Inhibitory control performance was assessed by the response time to Go stimuli (excluding response time $<100 \mathrm{~ms}$ and $<2$ or $>2$ standard-deviations to individual's mean RT) and by the false alarm rate to NoGo stimuli. In the expert group, we computed an index of the training load by dividing the total training time from the beginning of their practice (in minutes) by their age (in weeks).

\section{MRI data acquisition}

Data were acquired with a 3T MRI scanner (Discovery MR750; GE Healthcare, Waukesha, Wisconsin) with a 32-channel receive head coil. Stimuli were presented on an LCD screen (NordicNeuroLab, Bergen, Norway).

T1-weighted images were acquired with a FSPGR BRAVO sequence, voxel size: $0.86 \times 0.86 \times 1 \mathrm{~mm}$, number of coronal slices: $276, \mathrm{TR} / \mathrm{TE}=7300 / 2.8 \mathrm{~ms}$, flip angle $=9^{\circ}$, parallel imaging acceleration factor (PIAF): 1.5, intensity correction (SCIC).

Functional $T 2 *$ weighted echo planar images with blood oxygenation level-dependent (BOLD) contrast were acquired with: voxel size: $2.3 \times 2.3 \times 3 \mathrm{~mm}, 37$ ascending axial slices, inter-slice spacing $=0.2 \mathrm{~mm}, \quad \mathrm{TR} /$ $\mathrm{TE}=2000 / 30 \mathrm{~ms}$, Flip angle $=85^{\circ}$, PIAF: 2. A total of 552 volumes was acquired during the Go/NoGo and 447 during the 2-back (the last 34 volumes of each run corresponded to the tapping condition).

Diffusion tensor imaging (DTI) data was acquired using echo planar images with a voxel size of: $2 \times 2 \times 2 \mathrm{~mm}$, 60 axial slices, inter-slice spacing $=0.2 \mathrm{~mm}, \quad \mathrm{TR} /$ $\mathrm{TE}=8000 / 90.6 \mathrm{~ms}$, PIAF: 2, 30 non-collinear directions with $b$ value $=1000 \mathrm{~s} / \mathrm{mm}^{2}$, one $b=0$ image.

\section{Functional MRI}

We used the SPM8 software (Welcome Trust Centre for Neuroimaging, Institute of Neurology, University College London), running on Maltab 2012b (MathWorks, Inc., MA, USA) to analyze functional MRI data (fMRI). fMRI images were preprocessed following standard procedure (Friston et al. 2007). The fMRI preprocessing steps included a spatial realignment, slice timing (with middle temporal slice as reference), coregistration on T1 image, normalization on the Montreal Neurological Institute (MNI) space with $3 \times 3 \times 3 \mathrm{~mm}^{3}$ voxel size, and smoothing with a Gaussian kernel of 8 -mm full-width-athalf-maximum (FWHM). The preprocessed volumes were submitted to fixed effects analyses at the subject level by applying the general linear model to each voxel (Worsley and Friston 1995). Two separate models were built for the Go/NoGo and 2-back tasks; the "tapping" condition was included at the end of both models.

For the Go/NoGo, each stimulus onset was modeled as a delta function and convolved with the hemodynamic response function (HRF; Mechelli et al. 2003). Only the correct Go (hits) and NoGo (correct rejections) were considered in the analysis (misses and false alarms were modeled as conditions of no interest). In addition, movement parameters were included as regressors of no-interest. For the 2-back, stimuli were analyzed as blocks and convolved with the HRF. Movement parameters were not included since the 2-back model was designed as a block (Johnstone et al. 2006). The tapping condition was included as a block in both models. Time series from all voxels were submitted to a high-pass filter with a $1 / 250 \mathrm{~Hz}$ threshold, and an auto-regressive function (AR(1)) was applied.

We analyzed the MRI data using a region of interestbased approach (ROI) to increase our statistical power. Separate voxel-wise analyses for the 6 following AAL atlas ROIs (Tzourio-Mazoyer et al. 2002) were conducted: the right and left inferior frontal gyri (IFG); basal ganglia (BG; including the caudate, putamen and pallidum); and supplementary motor area (SMA). The ROIs were chosen based on (1) the ample literature pointing out these regions as the key nodes of the inhibitory control network (Aron et al. 2014); and (2) whole-brain Go/NoGo functional results confirming a critical role of the bilateral inferior frontal gyri in IC (Go vs NoGo fMRI contrast in the control and in the experts group).

The NoGo vs. Go $t$ contrast of each participant was submitted to a two samples $t$ test random effect model (RFX) to assess Expert vs. Controls group differences. To prevent motor activity related to the button press in Go but 
not in NoGo trials to bias the results, the activation of the tapping block was contrasted with the Go trials (Gotapping).

For the control 2-back task, we conducted a whole-brain analysis. The task vs. baseline t-contrast was computed for each participant and then submitted to an RFX two sample $t$ test to compare neural activity between the two groups. As for the previous model, the tapping block was subtracted from responses to the 2-back stimuli.

The analyses were conducted with the age and sex as regressors of no-interest.

For both experiments, the clusters' maxima reported were localized in the MNI and AAL atlas spaces with the WFU PickAtlas software (Maldjian et al. 2004; TzourioMazoyer et al. 2002). Results are displayed according to the neurological convention.

\section{Voxel-based morphometry}

Voxel-based morphometry (VBM, Ashburner and Friston 2000) on $T 1$-weighted images was performed using the procedure described in Ashburner (2009) with the SPM12 software. The VBM preprocessing steps were the following: $T 1$ images of each participant were segmented in grey matter (GM), white matter (WM) and cerebrospinal fluid (CSF). For optimal spatial precision we used in additional step the diffeomorphic spatial registration tool DARTEL (Ashburner 2007) followed by 12-parameter affine registration to the standardized Montreal Neurological Institute (MNI) space. GM probability maps were modulated to preserve relative volumes after spatial registration to MNI space. Finally, the resulting images were smoothed with an $8 \mathrm{~mm}$ FWHM isotropic Gaussian kernel. All grey matter volume (GMV) maps were then used in RFX models restricted to the ROIs.

Separate voxel-wise analyses (using two-sample $t$ test models) for each ROI were performed to test for GMV differences between the Expert vs Control group. The models included the age, total intracranial volume (TIV), and sex as regressors of no-interest. We used no grand mean scaling, no threshold masking, omitted global calculation, implicit, and explicit masks on the predefined ROI. A second VBM one-sample $t$ test analysis including only the Expert group was conducted to compute voxelwise correlations between GMV and index of the training load and behavioral performance (reaction time and number of false alarms), including TIV and age as regressors of no-interest. Separate statistical models were computed for each ROI (first and second analysis) and each index (second analysis). The significance threshold was set to $p_{\mathrm{FWE}}<0.05$ corrected for multiple comparisons at the voxel level for these analyses.
Tract-based spatial statistics (TBSS) of diffusion tensor imaging (DTI)

DTI data were analyzed with the TBSS approach (Smith et al. 2007) using the FSL 5.0.4 software (FMRIB software library, Jenkinson et al. 2012). The DTI data processing steps were the following: Diffusion-weighted images were affinealigned to the first b0 image using the eddy current correction of the FDT toolbox. A binary brain mask was generated, based on the b0 image, using BET tool with a 0.2 threshold. Next, the diffusion tensor was fitted to the data to compute the fractional anisotropy (FA) diffusion index (a measure of the relative levels of diffusion in different directions). As reported above, one of the experts previously included in the fMRI and VBM analysis was excluded from the TBSS analysis due to an abnormal BET mask of the b0 image.

The FA data were processed with the TBSS pipeline (Smith et al. 2007): nonlinearly transformed on the mean FA template (FMRIB58_FA) and then affine transformed on the standard MNI space. The resulting images were used to create the study-specific mean FA image which was skeletonized with a threshold FA $>0.2$ to generate the common white-matter tract skeleton map. Then, individual FA images were projected onto this reference skeleton.

To study the FA differences between the experts and the control participants, the processed data was analyzed using RFX two-sample $t$ tests including the age and sex as regressors of no-interest. In addition, an RFX one-sample $t$ test was conducted in the expert group to compute a voxel-wise correlation between white matter FA and the index of training load and behavioral performance, including age as a regressor of no-interest. As for the VBM analyses, the tests were performed separately and voxelwise within the ROIs (for first and second analysis) and for each index (second analysis). Statistical inference was based on the permuted p-values (5000 permutations; Nichols and Holmes 2002), which included the thresholdfree cluster enhancement (TFCE) with a threshold of $p<0.05$. The results were thickened to facilitate visualization. FA values at the clusters' maxima were extracted to produce scatterplots of FA against training intensity.

\section{Results}

\section{Behavior}

We tested the a priori hypothesis of a better IC in experts than controls by computing one-tailed independent sample $t$ tests on the behavioral indexes of performance: Experts' response times were significantly shorter than those of controls (mean \pm SEM, experts: $386.1 \mathrm{~ms} \pm 6.2$; controls: 
Fig. 1 Behavioral results. Inhibitory control proficiency was higher in the experts than in the control group, as indexed by faster response times to Go trials without concomitant increase in inhibition failures in the expert group. Asterisk $p<0.05$
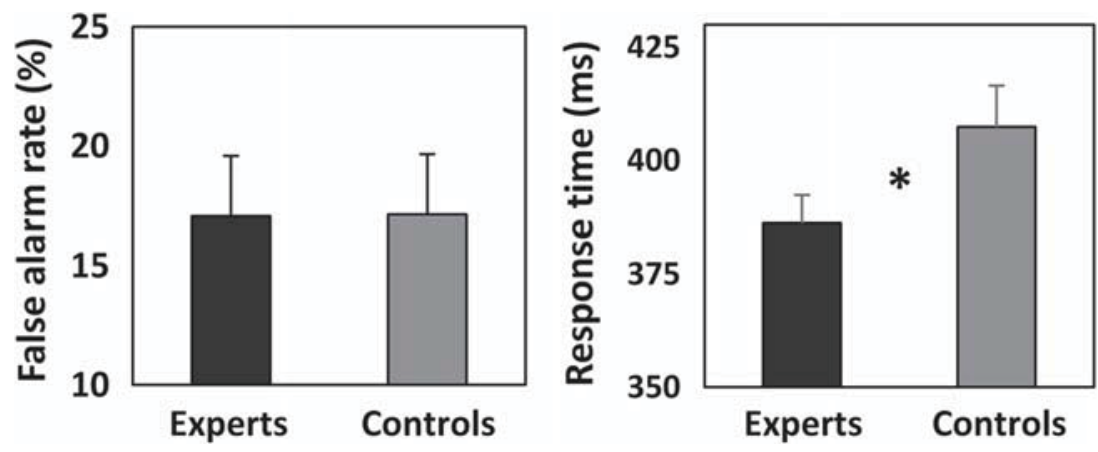

$407.4 \mathrm{~ms} \pm 9.1 ; t(35)=-1.95, p=0.029 ; \mathrm{Dz}=0.6)$ while there was no evidence for a different false alarm rate (mean \pm SEM, experts: $17.06 \% \pm 2.5$; controls: $17.13 \% \pm 2.5 ; t(35)=-0.19, p=0.49$; Fig. 1). There was no difference in the 2-back performance between the groups (RT and error rate; $p>0.2$ ).

\section{Functional magnetic resonance imaging}

Separated voxel-wise analyses comparing the experts' and the controls' neural activity during the Go/NoGo task within the right and left IFG, BG, and SMA ROIs revealed no significant differences between the two groups $\left(p_{\text {FWE }}<0.05\right.$; whole brain analyses did not reveal any differences, even at an uncorrected statistical threshold).

Whole brain analyses comparing the neural activity between the two groups during the 2-back task did not reveal any significant differences $\left(p_{\mathrm{FWE}}<0.05\right.$; the same ROI-based approach as for the Go/NoGo task did not reveal any difference).

\section{Grey matter: voxel-based morphometry}

The separate voxel-wise analyses comparing the experts and the controls within the six regions of interest revealed no significant GM volume difference $\left(p_{\mathrm{FWE}}<0.05\right.$ voxel level corrected).

Similarly, the correlations between the GM ROIs volume in the experts and the indexes of training and of behavioral performance at the IC task did not reach the $p_{\text {FWE }}<0.05$ voxel level corrected significance threshold. The same negative result was found when correlating GM volume (within the same ROIs as for the Go/NoGo task and whole brain) and behavioral performance in the 2-back control task as indexed by response time and percent error.

\section{White matter: diffusion tensor imaging tract-based spatial statistics (TBSS)}

The separate voxel-wise analyses comparing the fractional anisotropy (FA) between the experts and the controls within the six ROIs revealed higher FA within the right IFG ROI $(\mathrm{MNI} x y z=363310)$, left IFG ROI (MNI xyz $=-2417$ -18 ) and left BG ROI (MNI xyz $=-615-12)$ in the experts than in the control group $\left(p_{\mathrm{TFCE}}<0.05\right.$, cluster level corrected; Fig. 2). There was no evidence for higher FA in controls than in experts.

The correlations between the FA of the experts and the indexes of training and of behavioral performance revealed that the FA in the left BG correlated negatively $\left(p_{\mathrm{TFCE}}<0.05\right.$, cluster level corrected) with the response times to Go trials (MNI xyz $=-610-12$ ). In addition, the FA in the left SMA (intersection between the pre-SMA and the SMA proper) correlated positively with the total training load (MNI xyz $=-12-162$; Fig. 3).

There was no evidence for correlation between GM volume (within the same ROIs as for the Go/NoGo task and whole brain) and behavioral performance in the 2-back control task.

\section{Discussion}

Supranormal inhibitory control (IC) performance in the experts was associated with bilateral changes in the white matter microstructure of the inferior frontal gyrus. There were no concomitant alterations of grey matter volume or in the functional organization of inhibition-related networks. Behavioral performance in the inhibition task correlated with the fractional anisotropy (FA) of the basal ganglia white matter in the left hemisphere and the agenormalized total training load in the expert group correlated with the FA within the left supplementary motor area (SMA). As compared to the controls, the experts showed no behavioral superiority or functional difference during a control working memory 2-back task, indicating that their expertise was specific to inhibition.

Behaviorally, the effects of $20,000 \mathrm{~h}$ over 15 years of IC training mirrored those induced by short-term training regimens revealed in longitudinal IC training studies, namely a decrease in response speed to Go trials without concomitant increase in the rate of inhibition failure during the inhibition 
Fig. 2 White matter tract-based spatial statistics. Differences in white matter fractional anisotropy (FA) between the expert and the control group. Results are projected on the study-specific mean FA image with a TFCE corrected threshold of $p<0.05$. Results are thickened for visualization purpose. The study-specific skeleton is displayed in green. The bargraphs indicate the averaged FA values. MNI coordinate of the cluster maxima indicated

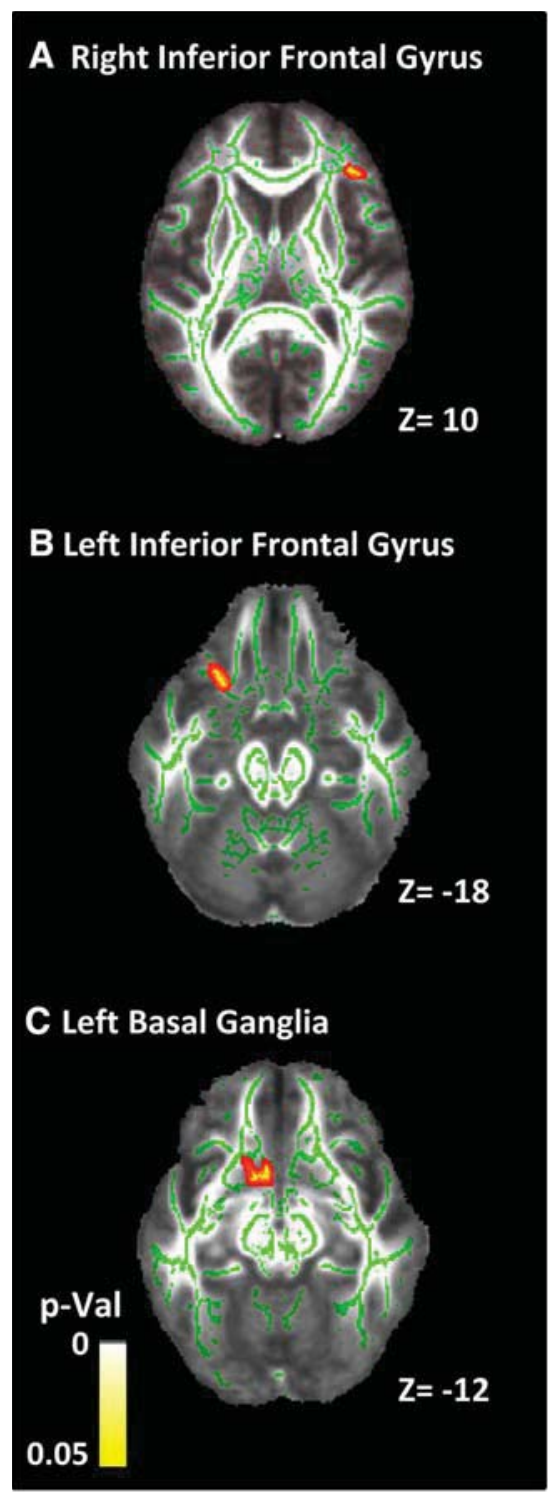

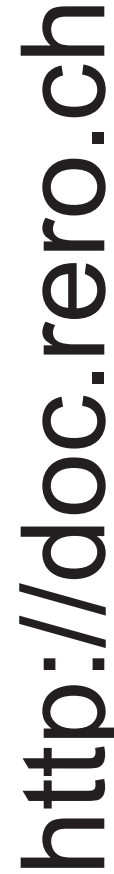

task. This pattern of behavioral improvement has been interpreted as reflecting an increase in the speed of inhibition processes based (1) on the fact that response speed during the inhibition task without increases in false alarm rate can only manifest if inhibition become faster (a mere improvement in response speed would result in an increase in false alarm rate via a speed-accuracy trade-off mechanism); and (2) on evidence that Go response speed correlates with the activity of regions involved in IC (IFG, medial frontal gyrus and BG; Benikos et al. 2013; Berkman et al. 2014; Chavan et al. 2015; Manuel et al. 2010, 2013; Verbruggen and 2012; White et al. 2014; Hartmann et al. 2015).

Our neurophysiological results support this account by revealing that changes in white matter microstructure within the fronto-basal IC network is the key mechanism for gaining long-term IC proficiency. As compared to the control group, the experts showed increases in FA within the left and right inferior frontal gyri, two key nodes of the IC network (Aron et al. 2014; Chavan et al. 2015; Hirose et al. 2012). Elevated white matter fractional anisotropy is thought to reflect changes in neurophysiological parameters which positively influence the speed at which action potentials spread along neural fibers, including myelination levels, axonal packing and axon diameters (Beaulieu 2002; Scholz et al. 2009).

Interestingly, we found no differences in the expert vs. the control group in the neural activity during the inhibition task, and no difference in grey matter morphometry. This finding contrasts with previous evidence for functional reorganizations of the inhibitory control network after short- to mediumterm training, which consistently associated decreases in the neural activity of the IFG to inhibition trials with 
Fig. 3 White matter regression analyses. There was a

significant negative correlation between the left basal ganglia (BG) white matter fractional anisotropy (FA) of the experts and their $\mathrm{Go} / \mathrm{NoGo}$ response times (in milliseconds; a) and a positive correlation between the FA of the left supplementary motor area (SMA) and the total training load (in minutes per weeks of life; b). MNI coordinates of the cluster maxima are indicated
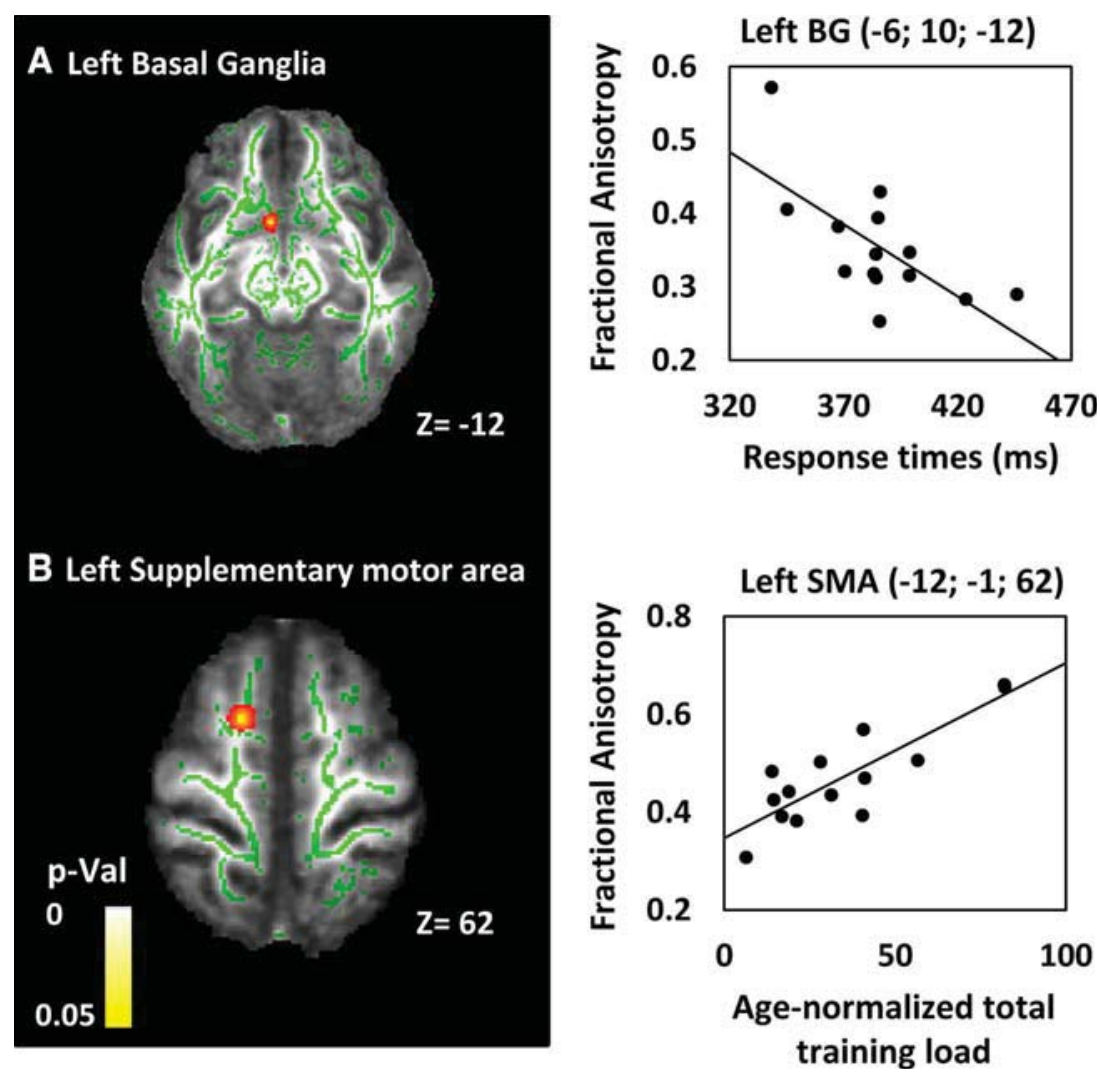

improvements in IC proficiency [e.g., (Chavan et al. 2015; Hartmann et al. 2015)]. Although negative results should be interpreted with caution, these results suggest that the BOLD and VBM effect sizes induced by 15 years of intensive IC training were smaller than those induced by short-term training because in Chavan et al. (2015), the same analytical procedure managed to reveal plastic modifications induced by only 3 weeks of training in a sample of a comparable size. According to the hypothesis on the effect of IC training mentioned above, one could also advance that there were functional differences between the two groups, but only at the level of the temporal dynamic of the inhibition process. Since such differences would have manifested in the millisecond range, the classical fMRI analyses used in the present study would have had a too low temporal resolution to reveal them.

Further supporting the key role of white matter microstructure in sustained improvements in IC proficiency, we found a negative correlation between response times during the Go/NoGo task and the FA adjacent to basal ganglia (BG). The BG constitute the target of the projection from the inferior frontal gyrus within the IC network and mediate inhibition via their projections to primary motor areas (Aron et al. 2014). There was also a positive correlation between the FA at the intersection between the left SMA and pre-SMA and the age-normalized total training load. The SMA has been involved in motor execution (Lee et al. 1999; Nachev et al. 2007) and the pre-SMA in the control of impulse and, notably, in the inhibition of their behavioral expression (Herz et al. 2014; Spieser et al. 2015). Accordingly, the training might have reinforced the interaction between these two regions to reach faster movement control. The left-lateralization of these two effects likely follows from the fact that the experts were all right-handed.

Importantly, while we did not assess directly if the fencers also showed improved motor execution or selection in addition to motor inhibition, that was most likely the case and differences at this level with the control group could also partly account for our pattern of results. Our finding for a correlation between the amount of training and the SMA FA could have followed from an effect of fencers' training on motor execution, this region being associated with such processes (e.g., Simmonds et al. 2008). In the same vein, the inhibitory control regions of interest in the present study have likewise been involved in response planning and selection (e.g., Mostofsky and Simmonds 2008).

In spite of these correlations between the levels of expertise and the structural variables, and since our control group did not participate in an intensive training unrelated to inhibitory control, we cannot rule out that genetic factors were at the origin of the differences observed between these two groups. One could indeed advance that there was a selection bias in the 
expert group based on a specific neural architecture favoring either IC proficiency, or the self-discipline necessary to follow an intensive training regimen over several years. Longitudinal studies may help disentangling this question.

Together with previous literature, our collective results show that, while phasic changes in functional and grey matter architectures accompany IC behavioral improvement after short-term training, prominently white matter modifications are involved in long-lasting IC improvements.

Acknowledgments This work was supported by grants from the Swiss National Science Foundation (Grant \#32003B_156854 to LS and \#31003A_153070 to WZ). BD is supported by the Swiss National Science Foundation (NCCR Synapsy, project grant \#320030_135679 and SPUM 33CM30_140332/1), Foundation Parkinson Switzerland and Foundation Synapsis. The research leading to these results has received funding from the European Union Seventh Framework Programme (FP7/2007-2013) under grant agreement no. 604102 (Human Brain Project). LREN is very grateful to the Roger de Spoelberch and Partridge Foundations for their generous financial support.

\section{References}

Aron AR, Robbins TW, Poldrack RA (2014) Inhibition and the right inferior frontal cortex: one decade on. [Research Support, N.I.H., Extramural Research Support, Non-US Gov't]. Trends Cogn Sci 18(4):177-185. doi:10.1016/j.tics.2013.12.003

Ashburner J (2007) A fast diffeomorphic image registration algorithm. Neuroimage 38(1):95-113

Ashburner J (2009) Computational anatomy with the SPM software. [Research Support, Non-US Gov't Review]. Magn Reson Imaging 27(8):1163-1174. doi:10.1016/j.mri.2009.01.006

Ashburner J, Friston KJ (2000) Voxel-based morphometry-the methods [Research Support, Non-US Gov't Review]. Neuroimage 11(6 Pt 1):805-821. doi:10.1006/nimg.2000.0582

Beaulieu C (2002) The basis of anisotropic water diffusion in the nervous system-a technical review. [Research Support, Non-US Gov't Review]. NMR Biomed 15(7-8):435-455. doi:10.1002/nbm.782

Benikos N, Johnstone SJ, Roodenrys SJ (2013) Short-term training in the Go/Nogo task: behavioural and neural changes depend on task demands. Int J Psychophysiol 87(3):301-312. doi:10.1016/j. ijpsycho.2012.12.001

Berkman ET, Kahn LE, Merchant JS (2014) Training-induced changes in inhibitory control network activity. [Randomized Controlled Trial Research Support, Non-US Gov't]. J Neurosci 34(1):149-157. doi:10.1523/JNEUROSCI.3564-13.2014

Chan JSY, Wong AC-N, Liu Y, Yu J, Yan JH (2011) Fencing expertise and physical fitness enhance action inhibition. Psychol Sport Exerc 1212(5):509-514

Chavan CF, Mouthon M, Draganski B, van der Zwaag W, Spierer L (2015) Differential patterns of functional and structural plasticity within and between inferior frontal gyri support training-induced improvements in inhibitory control proficiency. Hum Brain Mapp. doi:10.1002/hbm.22789

Di Russo F, Taddei F, Apnile T, Spinelli D (2006) Neural correlates of fast stimulus discrimination and response selection in toplevel fencers. [Research Support, Non-US Gov't]. Neurosci Lett 408(2):113-118. doi:10.1016/j.neulet.2006.08.085

Friston K, Mattout J, Trujillo-Barreto N, Ashburner J, Penny W (2007) Variational free energy and the Laplace approximation [Research Support, Non-US Gov't]. Neuroimage 34(1):220-234. doi:10.1016/j.neuroimage.2006.08.035
Hartmann L, Sallard E, Spierer L (2015) Enhancing frontal top-down inhibitory control with Go/NoGo training. Brain Struct Funct [Epub ahead of print] PMID: 26459141

Herz DM, Christensen MS, Bruggemann N, Hulme OJ, Ridderinkhof KR, Madsen KH, Siebner HR (2014) Motivational tuning of fronto-subthalamic connectivity facilitates control of action impulses. J Neurosci 34(9):3210-3217. doi:10.1523/JNEUR OSCI.4081-13.2014

Hirose S, Chikazoe J, Watanabe T, Jimura K, Kunimatsu A, Abe O, Konishi S (2012) Efficiency of go/no-go task performance implemented in the left hemisphere. [Research Support, Non-US Gov't]. J Neurosci 32(26):9059-9065. doi:10.1523/JNEUR OSCI.0540-12.2012

Jenkinson M, Beckmann CF, Behrens TE, Woolrich MW, Smith SM (2012) Fsl [historical article review]. Neuroimage 62(2):782-790. doi:10.1016/j.neuroimage.2011.09.015

Johnstone T, Ores Walsh KS, Greischar LL, Alexander AL, Fox AS, Davidson RJ, Oakes TR (2006) Motion correction and the use of motion covariates in multiple-subject fMRI analysis. [Research Support, N.I.H., Extramural]. Hum Brain Mapp 27(10):779-788. doi: $10.1002 / \mathrm{hbm} .20219$

Lee KM, Chang KH, Roh JK (1999) Subregions within the supplementary motor area activated at different stages of movement preparation and execution. Neuroimage 9(1):117-123

Maldjian JA, Laurienti PJ, Burdette JH (2004) Precentral gyrus discrepancy in electronic versions of the Talairach atlas. Neuroimage 21(1):450-455

Manuel AL, Grivel J, Bernasconi F, Murray MM, Spierer L (2010) Brain dynamics underlying training-induced improvement in suppressing inappropriate action. [Research Support, Non-US Gov't]. J Neurosci 30(41):13670-13678. doi:10.1523/JNEUR OSCI.2064-10.2010

Manuel AL, Bernasconi F, Spierer L (2013) Plastic modifications within inhibitory control networks induced by practicing a stop-signal task: an electrical neuroimaging study [Research Support, Non-US Gov't]. Cortex 49(4):1141-1147. doi:10.1016/j.cortex.2012.12.009

Mechelli A, Henson RN, Price CJ, Friston KJ (2003) Comparing event-related and epoch analysis in blocked design fMRI. Neuroimage 18(3):806-810

Mostofsky SH, Simmonds DJ (2008) Response inhibition and response selection: two sides of the same coin. J Cogn Neurosci 20(5):751-761. doi:10.1162/jocn.2008.20500

Nachev P, Wydell H, O'neill K, Husain M, Kennard C (2007) The role of the pre-supplementary motor area in the control of action. Neuroimage 36(Suppl 2):T155-T163

Nichols TE, Holmes AP (2002) Nonparametric permutation tests for functional neuroimaging: a primer with examples. [Research Support, Non-US Gov't]. Hum Brain Mapp 15(1):1-25

Oldfield RC (1971) The assessment and analysis of handedness: the Edinburgh inventory. Neuropsychologia 9(1):97-113

Roi GS, Bianchedi D (2008) The science of fencing: implications for performance and injury prevention [Review]. Sports Med 38(6):465-481

Scholz J, Klein MC, Behrens TE, Johansen-Berg H (2009) Training induces changes in white-matter architecture. [Research Support, Non-US Gov't]. Nat Neurosci 12(11):1370-1371. doi:10.1038/ nn. 2412

Simmonds DJ, Pekar JJ, Mostofsky SH (2008) Meta-analysis of Go/ No-go tasks demonstrating that fMRI activation associated with response inhibition is task-dependent. Neuropsychologia 46(1):224-232

Smith SM, Johansen-Berg H, Jenkinson M, Rueckert D, Nichols TE, Miller KL, Behrens TE (2007) Acquisition and voxelwise analysis of multi-subject diffusion data with tract-based spatial statistics. [Research Support, Non-US Gov't]. Nat Protoc 2(3):499-503. doi:10.1038/nprot.2007.45 
Spierer L, Chavan CF, Manuel AL (2013) Training-induced behavioral and brain plasticity in inhibitory control. Front Hum Neurosci 7:427. doi:10.3389/fnhum.2013.00427

Spieser L, van den Wildenberg W, Hasbroucq T, Ridderinkhof KR, Burle B (2015) Controlling your impulses: electrical stimulation of the human supplementary motor complex prevents impulsive errors. J Neurosci 35(7):3010-3015. doi:10.1523/JNEUROSCI. 1642-14.2015

Taddei F, Bultrini A, Spinelli D, Di Russo F (2012) Neural correlates of attentional and executive processing in middle-age fencers. [Research Support, Non-US Gov't]. Med Sci Sports Exerc 44(6):1057-1066. doi:10.1249/MSS.0b013e31824529c2

Tuch DS, Salat DH, Wisco JJ, Zaleta AK, Hevelone ND, Rosas HD (2005) Choice reaction time performance correlates with diffusion anisotropy in white matter pathways supporting visuospatial attention. Proc Natl Acad Sci USA 102(34):12212-12217. doi:10.1073/pnas.0407259102

Tzourio-Mazoyer N, Landeau B, Papathanassiou D, Crivello F, Etard O, Delcroix N, Joliot M (2002) Automated anatomical labeling of activations in SPM using a macroscopic anatomical parcellation of the MNI MRI single-subject brain. Neuroimage 15(1):273-289. doi:10.1006/nimg.2001.0978

Verbruggen F, Adams R, Chambers CD (2012) Proactive motor control reduces monetary risk taking in gambling. [Research Support, Non-US Gov't]. Psychol Sci 23(7):805-815. doi:10. $1177 / 0956797611434538$

Waxman SG (1980) Determinants of conduction velocity in myelinated nerve fibers. Muscle Nerve 3:141-150

White CN, Congdon E, Mumford JA, Karlsgodt KH, Sabb FW, Freimer NB, Poldrack RA (2014) Decomposing decision components in the stop-signal task: a model-based approach to individual differences in inhibitory control. [Research Support, N.I.H., Extramural Research Support, Non-US Gov't]. J Cogn Neurosci 26(8):1601-1614. doi:10.1162/jocn_a_00567

Worsley KJ, Friston KJ (1995) Analysis of fMRI time-series revisited-again. [Comment Research Support, Non-US Gov't]. Neuroimage 2(3):173-181. doi:10.1006/nimg.1995.1023 